\title{
False Color Method for Retinal Oximetry
}

\section{Erwin Michel Davila-Iniesta1 ${ }^{1}$, Santiago Guerrero-Gonzalez ${ }^{1}$, Jorge Santiago-Amaya1, Paola Castillo-Juarez ${ }^{2}$, Luis Niño-de-Rivera-Oyarzabal ${ }^{1}$}

${ }^{1}$ SEPI ESIME Culhuacan, Instituto Politecnico Nacional, Mexico City, Mexico; ${ }^{2}$ ENCB Santo Tomas, Instituto Politecnico Nacional, Mexico City, Mexico

Correspondence to: Luis Niño-de-Rivera-Oyarzabal, luisninoderivera@gmail.com

Keywords: Choroidal Structure, False Color Method, Filters, Fundus, OCT Image, Oxygen Saturation, Oximetry, Retinal Vessels

Received: October 15, $2019 \quad$ Accepted: December 17, $2019 \quad$ Published: December 20, 2019

Copyright (c 2019 by author(s) and Scientific Research Publishing Inc.

This work is licensed under the Creative Commons Attribution International License (CC BY 4.0).

http://creativecommons.org/licenses/by/4.0/

\section{(c) (i) Open Access}

\section{ABSTRACT}

Oximetry is a method for measuring the oxygen saturation of haemoglobin in blood. Particularly, retinal oximetry based in the measurement of oxygen saturation in retinal vessels has acquired great interest to gather information on blood oxygenation from said vessels within inner and outer retina. Non-invasive spectrophotometric retinal oximetry has been studied for over five decades based on imaging spectroscopy. However, Optical Coherence Tomography (OCT) is an alternative to analyze the absorption difference between oxyhaemoglobin $\left(\mathrm{HbO}_{2}\right)$ and deoxyhaemoglobin $(\mathrm{Hb})$ in the retinal vessels and the choroidal structure. We propose in this paper an alternative process to manipulate conventional OCT images to evaluate changes in the relative haemoglobin oxygen saturation. Conventional OCT images from $570 \mathrm{~nm}$ and $600 \mathrm{~nm}$ in gray scale are converted to a corresponding color scale to be compared to the oxygenation information involved in the original gray scale OCT images.

\section{INTRODUCTION}

Fundus images are obtained by an internal view of the eye through the pupil and the transparent means lodged in the eyeball and the retina (namely cornea, crystalline, aqueous humor and vitreous humor) [1]. The study is performed by projecting a beam of light into the pupil to facilitate the visualization of the fundus. Fundus images can determine if a person has an eye condition, which could be retinal detachment, retinal thrombosis, macular degeneration, diabetic retinopathy, glaucoma, as well as being able to locate said condition [2]. In order to obtain a fundus image indirect observation is used, done by means of a slit lamp, in which the patient must remain motionless, sitting in a straight position and with their chin resting on a base approximately $15 \mathrm{~cm}$ away from the lamp. Their eye must remain open while 
light reflects inside the walls of the eye so a three-dimensional reconstruction of the interior can be performed. The clarity of the image depends directly on the frequency that the image is obtained, which comprises a range between 570 and $600 \mathrm{~nm}$ [3].

On the other hand, oxymetry is the study of oxygen saturation measurement within the ocular vasculature. In our case study, oxymetry is based on oxyhaemoglobin $\left(\mathrm{HbO}_{2}\right)$ and deoxyhaemoglobin $(\mathrm{Hb})$. Concerning the obtaining of the amount of oxygen within the blood vessels it is necessary to take advantage of the properties within $\mathrm{HbO}_{2}$ and $\mathrm{Hb}$ when absorbing light. Additionally, it is imperative to determine the percentage of $\mathrm{HbO}_{2}$, which is known as oxygen saturation. Measuring oxygen saturation helps to know the state of the retina. At the time of this study, a beam of light with wavelengths ranging from 500 to $700 \mathrm{~nm}$ is applied, depending on the study performed [4]. These studies have aroused interest in finding the vasculature and detecting oxygen saturation inward the fundus, especially in the retina, since these are non-invasive techniques, therefore not representing any threat for human health [5].

\section{METHODOLOGY}

The fundus image shown in Figure 1 [6] displays an internal visualization of the eyeball structure through an image taken from the transparent media contained within the retina mentioned in the Introduction. Retinal vessels are the only blood vessels in the human body that can be observed directly through the pupil of the eye.

Figure 2 presents the light absorption capacity between $\mathrm{Hb}$ and $\mathrm{HbO}_{2}$ varies with the wavelenght of the beam of light, giving out ranges between 500 and $640 \mathrm{~nm}$ for both $\mathrm{Hb}$ and $\mathrm{HbO}_{2}$. Please note that 570 and $600 \mathrm{~nm}$ are two key wavelengths in the frequency response of $\mathrm{Hb}$ and $\mathrm{HbO}_{2}$. This property allows us to find any differences between the $\mathrm{Hb}$ and $\mathrm{HbO}_{2}$. Jóna Valgerður Kristjánsdóttir described the results of

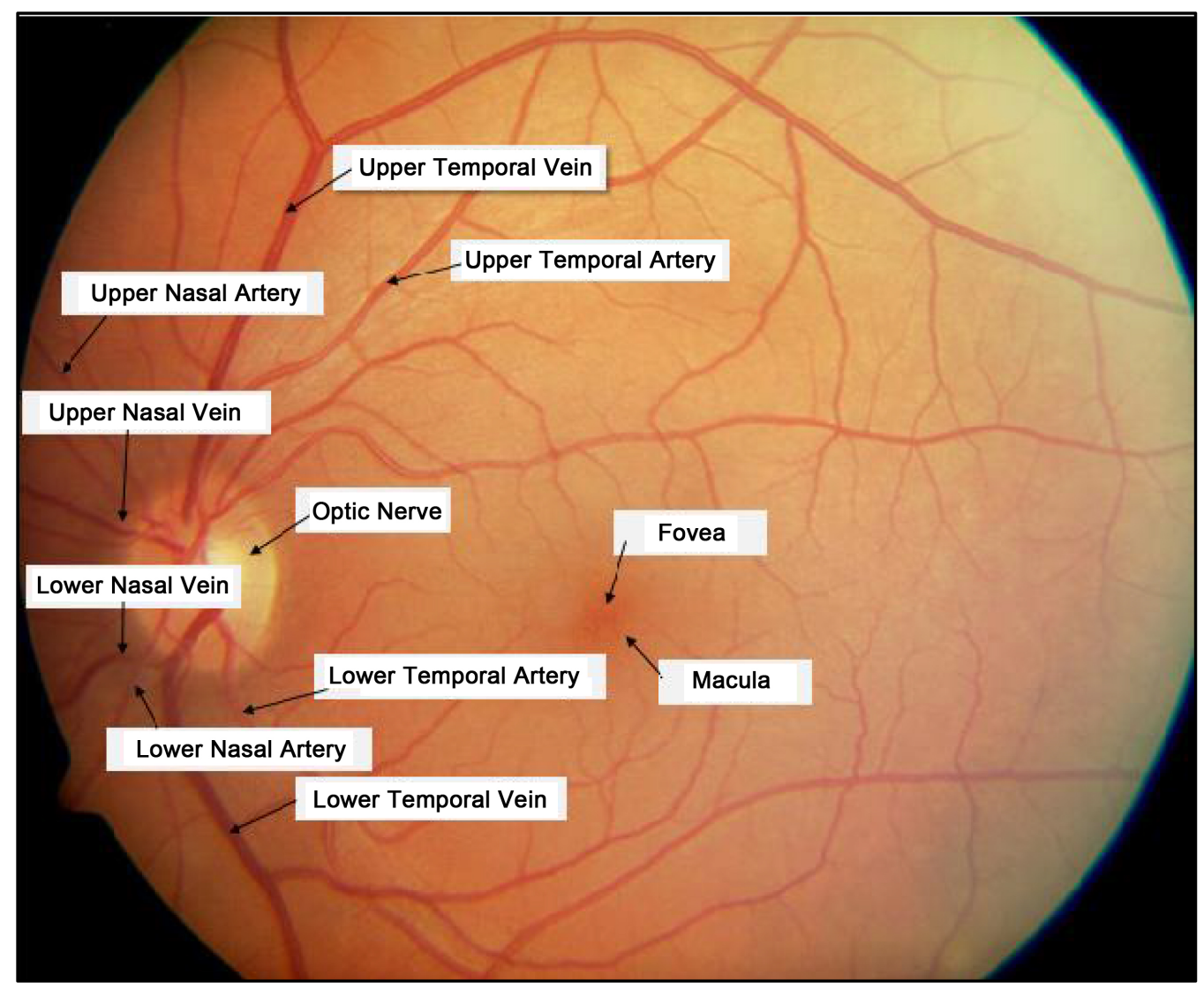

Figure 1. Eyeball structure visualized in a fundus image. 


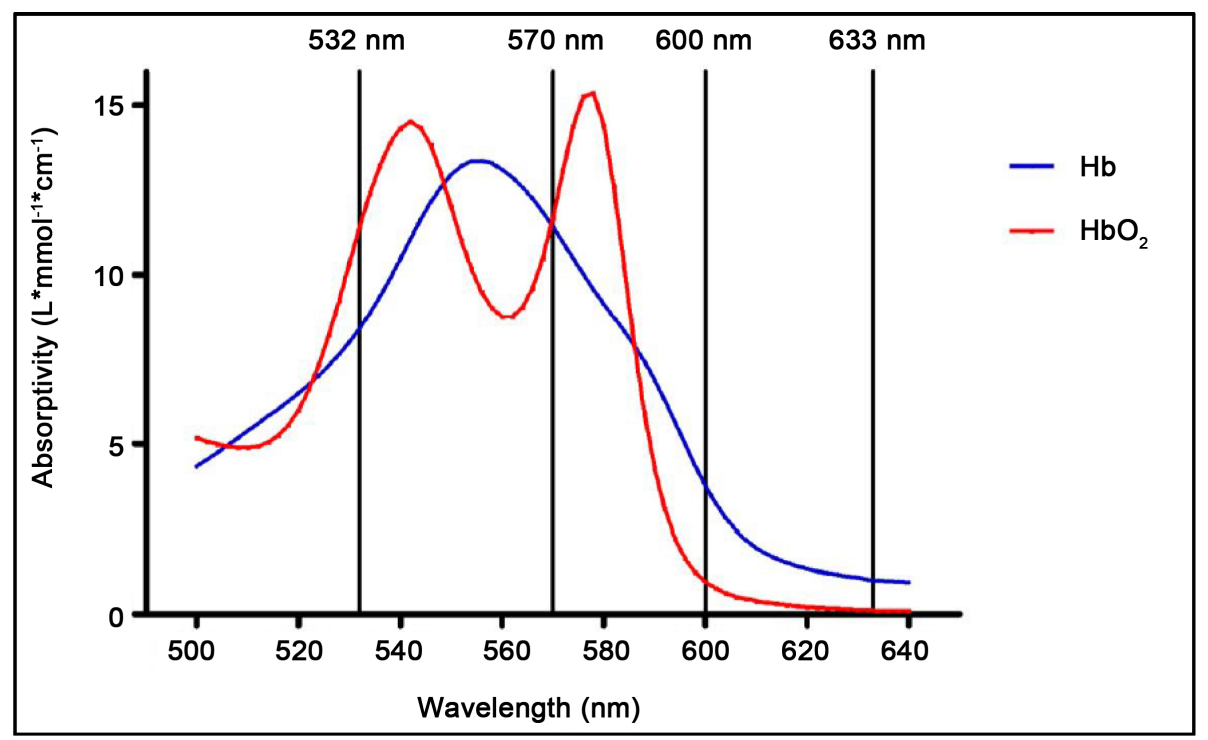

Figure 2. Light absorptivity of oxyhaemoglobin $\left(\mathrm{HbO}_{2}-\right.$ continuous red line) and deoxyhaemoglobin ( $\mathrm{Hb}$-continuous blue line). Light absorptivity for $\mathrm{Hb}$ and $\mathrm{HbO}_{2}$ is the same at isosbestic wavelength (i.e. $570 \mathrm{~nm}$ ) but different at non-isosbestic wavelengths (i.e. $600 \mathrm{~nm}$ ).

light absorption capacity in her study [3]. $\mathrm{HbO}_{2}$ oxygen saturation is usually measured using non-invasive spectrophotometric oximetry based on $\mathrm{HbO}_{2}$ and $\mathrm{Hb}$ having different light absorption properties, i.e. a different color. Therefore, deoxygenated blood (less $\mathrm{HbO}_{2}$ ) has a dark red color and fully oxygenated blood (more $\mathrm{HbO}_{2}$ ) has a brighter red color [7].

Fundus images in which some filters are applied may contain details and damaged areas that are unnoticeable to the naked eye [8]. Comparison in digital images can be used as a filter, visualizing its displacement and the deformations inside it along a determined period of time. With this method we can find how similar one image is to another, depending on the intensity in the shades of gray allocated in the image.

As a first approach, in order to detect the minimal number of variations in the vessels and the tissues of the retina, we proposed to compare a fundus image obtained by means of an OCT with itself but changing it in one pixel [9]. This allows us to show that such differences of a pixel are detected, as shown in Figure 6 and Figure 7. In this method, the original fundus image is taken and converted to a grayscale image, consisting of 256 shades of gray. Therefore, we obtained an image that contains a minimal amount of noise, thus avoiding any alteration through any study. A low-pass filter was designed, which takes the form of the Pascal Triangle, avoiding Gaussian noise, as shown in Figure 3.

In Figure 4 we used the same low-pass filter as a contrast enhancement to increase the amount of white in the image to highly detail some features that are unnoticeable when the image is displayed in grayscale. Therefore, we obtained a negative image to simulate the disc angiography images [10], displayed in Figure 5.

The results shown in Figures 3-5 were compared with the original OCT image that was digitally modified to make the relevant comparison. This process consisted of applying the same treatment that received the original OCT image and then being able to compare the image with respect to it. This was achieved by comparing a pixel at a certain position with its similar artificial modification image. If the pixels between them have the same shade of gray, the same pixel will be displayed. On the other hand, if the pixel in the original image is different from that of the modified image, the proposed method automatically subtracts it between the values that the pixels have, showing the hue obtained between the subtractions 


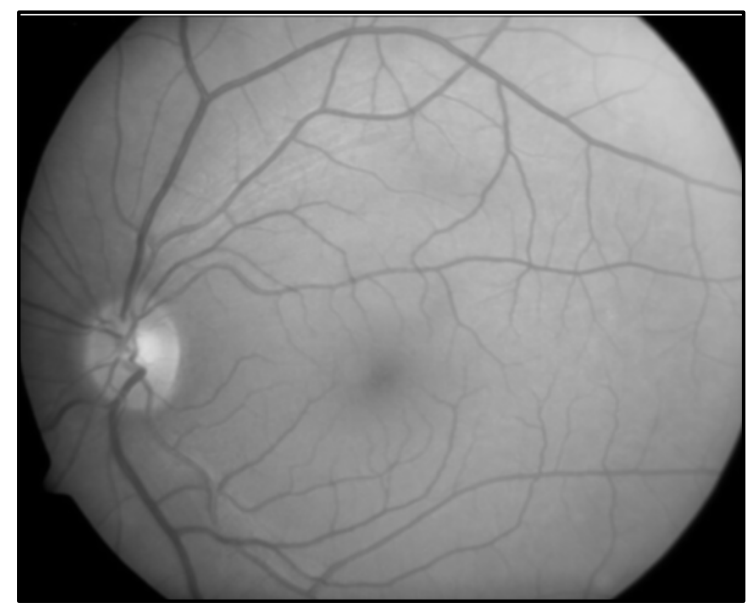

Figure 3. Low-pass filter applied to the original fundus image.

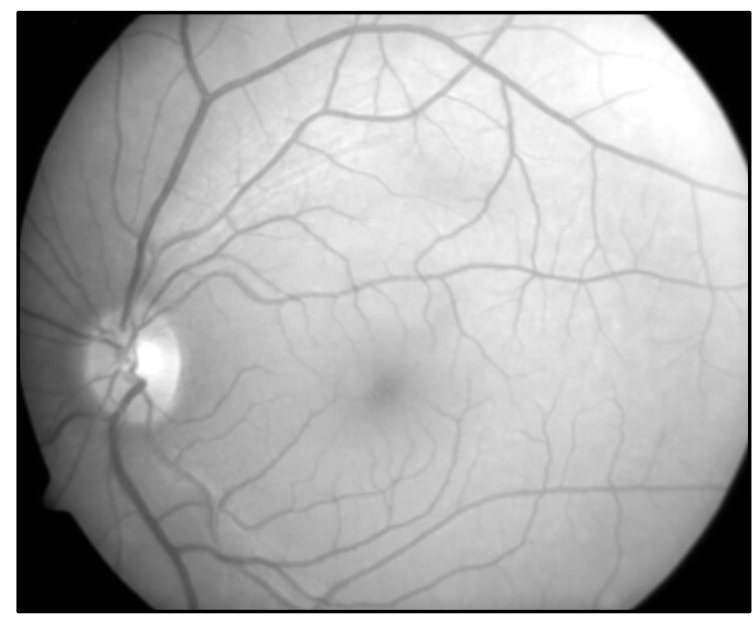

Figure 4. Contrast enhancement applied to the filtered fundus image.

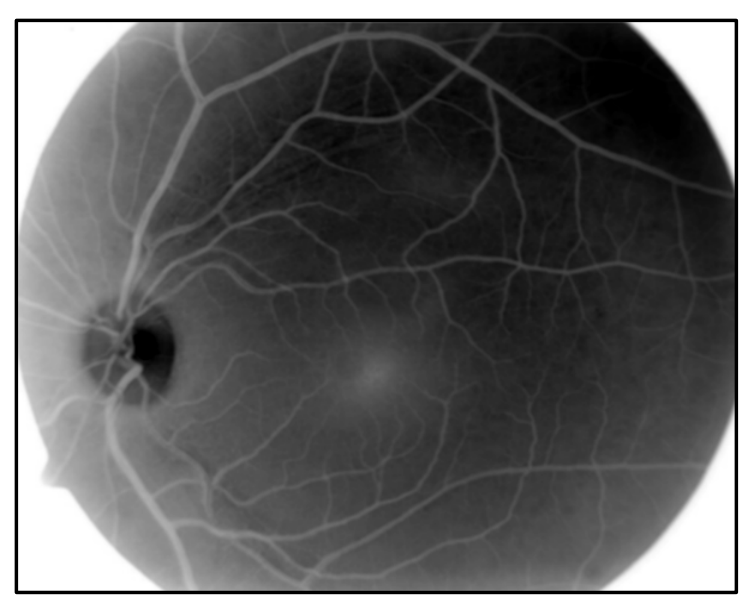

Figure 5. Negative of the fundus image with contrast enhancement. 
of these values.

We randomly selected one of the two images that were initially compared in order to apply the False Color Method. We apply the Top-Hat and Bottom-Hat filters between the histogram equalization with the intention of highlighting the details that are not visible in the original OCT image to enhance the selected image [11]. Therefore, the image resulting from the contrast improvement was equalized to observe 16 gray intensities within the image, thus the False Color Method can be applied to visualize the oxygenation of the structure on a new color scale. This new color scale clearly displays the differences between $\mathrm{Hb}$ and $\mathrm{HbO}_{2}$, as seen in Figure $2(570$ and $600 \mathrm{~nm})$.

\section{RESULTS}

The results in Figure 6 show the differences between the original OCT image and its modified counterpart, as previously stated in the Methodology section. These differences consist of small black dots due to subtraction between the values of the shades of gray found inside the images. It is important to mention that the black dots in the final image represent a minimal amount of noise that still remains in the image after applying the low-pass filter to eliminate said noise.

In order to obtain the Region of Interest (ROI), we need to apply a cut, called trimming, to the image resulting from comparing both images, shown in Figure 7. This allows us to determine which area presents differences when being analyzed throughout the study.

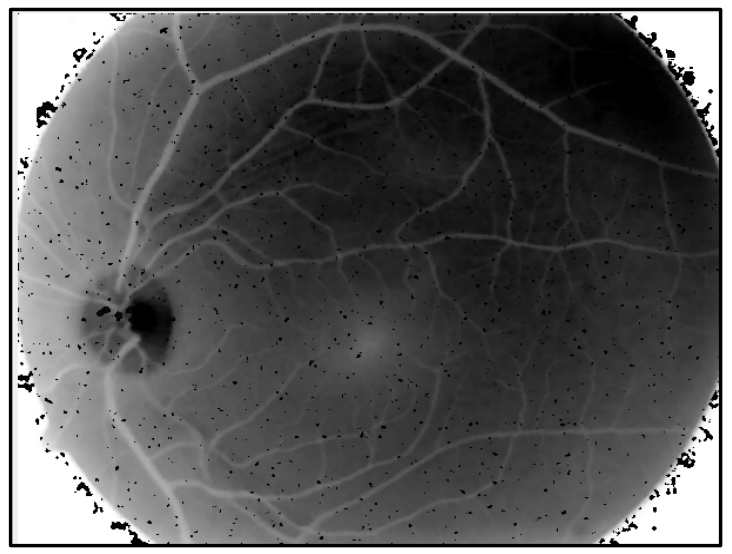

Figure 6. Resulting fundus image from the comparison between the original and the modified OCT images.

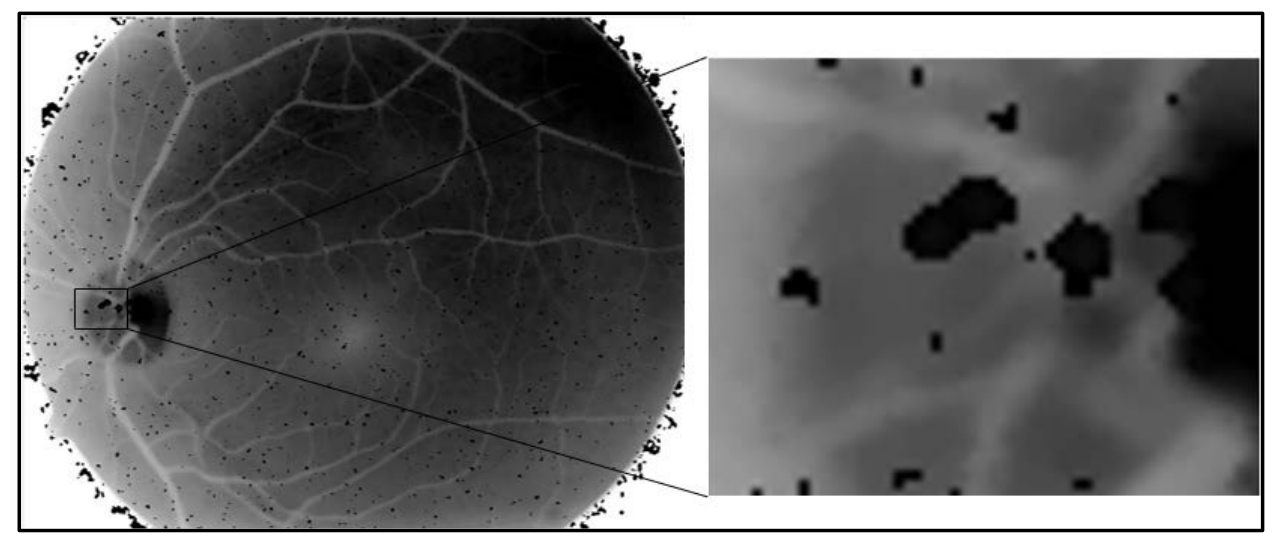

Figure 7. Trimming of the compared fundus image. 
When the compared fundus image is trimmed, the developed algorithm applied to the fundus images and the False Color Method for retinaoxymetry makes the distinction of the tone of the grays in the image. Consequently, the False Color Method is applied to one of the two images that were selected earlier. In this case, Figure 3 was taken into account, now called Figure 8(A), which was divided into three parts: Red, Green and Blue (Figures 8(B)-(D), respectively). Once all the three images were obtained, we were given the option to select any of these images. In our case study, we selected the green image, as it is the image that gives out more information.

In Figure 8(E), the Bottom-Hat filter was applied, obtaining a new image highlighting some regions of the image displayed in Figure 8(C). After applying the Top-Hat filter, a contrast enhancement was applied to further lighten the image obtained with said filter. Subsequently, the histogram was equalized to take into account only 16 shades of gray, with the intention of minimizing the amount of intensities to be processed in the False Color Method algorithm.

We show in Figure 9(D) the corresponding fundus image once the False Color Method was applied.

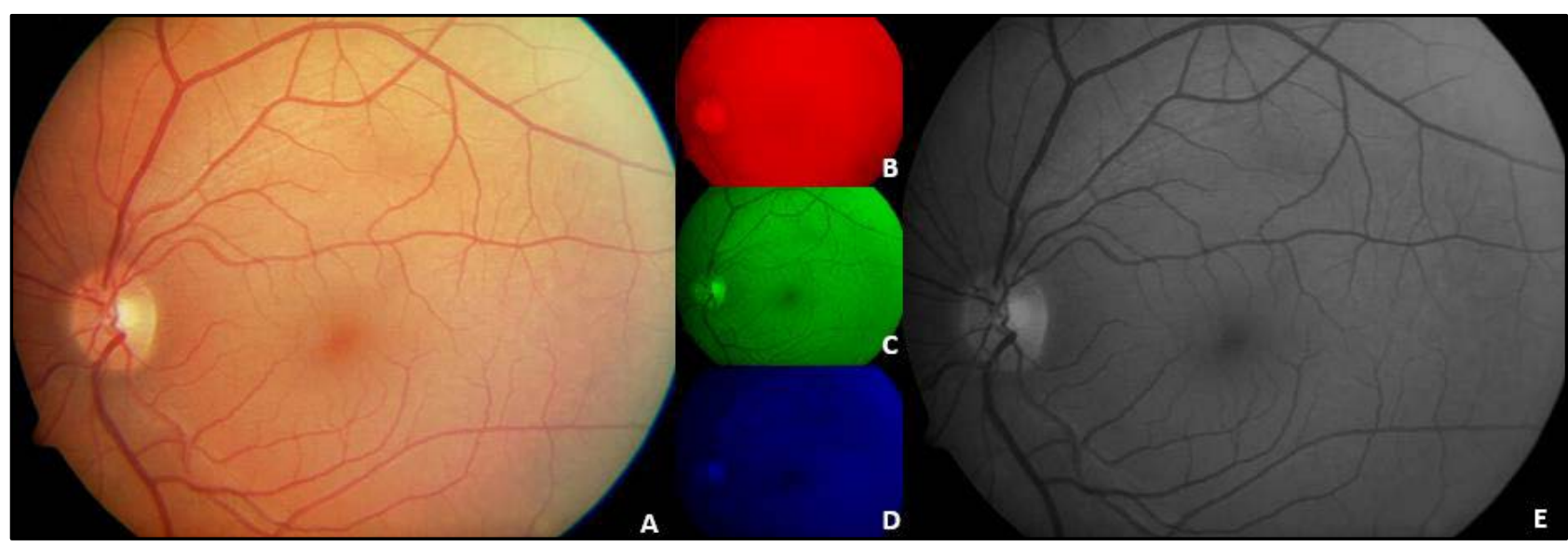

Figure 8. (A) Original fundus image of a healthy eye. (B) Red Image. (C) Green image. (D) Blue image. (E) Image 8-C after a low-pass filter was applied, resulting in the obtaining of the grayscale image.

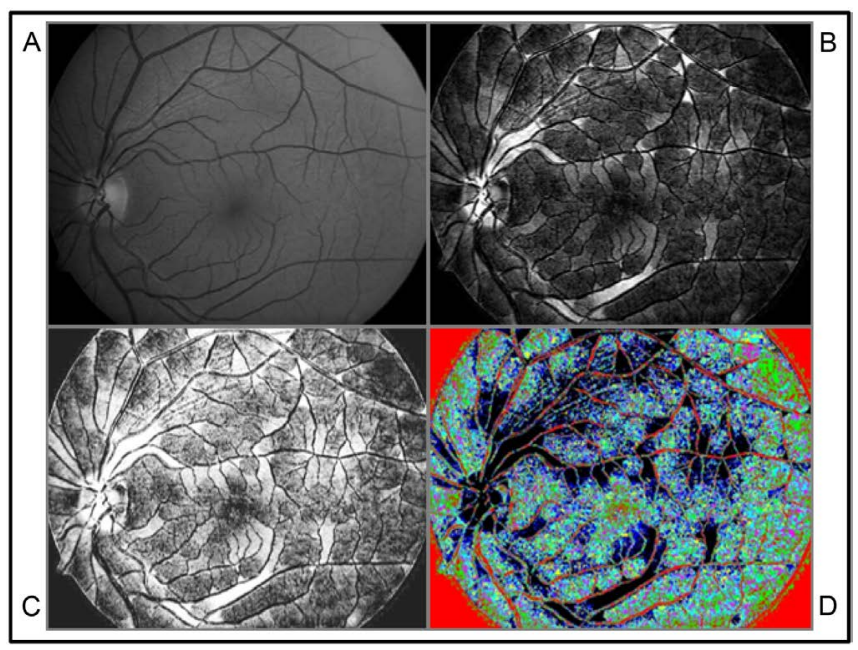

Figure 9. (A) Image with Bottom-Hat filter application. (B) Contrast enhancement to image 12-A. (C). Histogram equalization with 16 shades of gray to Image 12-B. (D) False color method APPLIED to image 12-C. 
Oxygen saturation in blood vessels is represented by internal colors, where red represents maximum oxygen saturation, green is intermediate saturation, and blue null saturation. The proposed software automatically identifies the blood vessels and selects points without illumination from the images, which correspond to the maximum absorptivity at $600 \mathrm{~nm}$; case contrary at $570 \mathrm{~nm}$, according to Figure 2. After identifying these places, oxygen saturation is calculated and represented by colors, so that the red color represents the maximum oxygen saturation, the green color intermediate oxygen saturation and the blue color null oxygen saturation. Color adjustment must be done according to $570 \mathrm{~nm}$. As can be seen in Figure 9(C), a dark grayscale will result in a brighter False Color Method image. However, the color allocation conditions are not well established, since this is a novel study.

Please note that Figure 8(A) corresponds to an image of a healthy eye, obtained from [12]. From here, Figure $8(B)$ to Figure 10 correspond to the application of the proposed method to the same image, as explained previously.

Figure 13 shows the detailed image from Figure 12(D). As explained before, Figure 11(C) gives out the most amount of information to get the grayscale image shown in Figure 11(E). Once this step is done, the filters in Figure 9 are applied. The results are displayed in Figure 12 showing an important enhancement to the original OCT image from Figure 12(A).

It is worth mentioning that Figure 11(A) was obtained from [6]. Subsequently, Figure 11(B) to Figure 13 shows the aforementioned method applied.

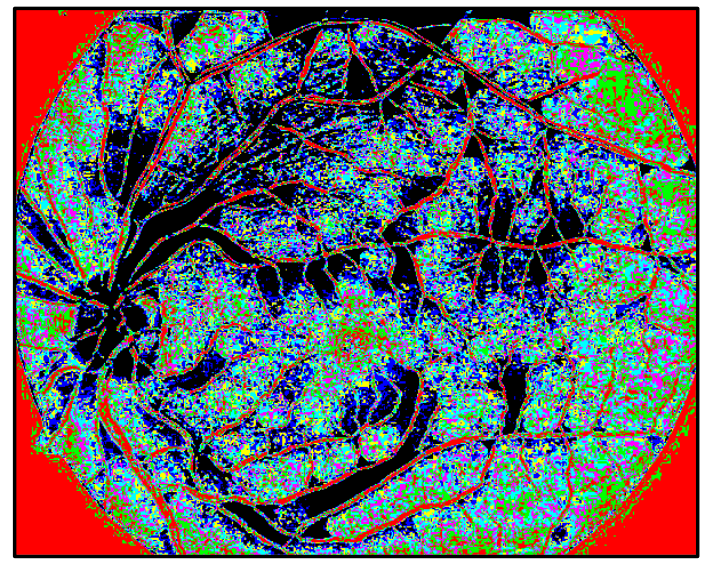

Figure 10. False Color Method with the oxygen saturation specification.

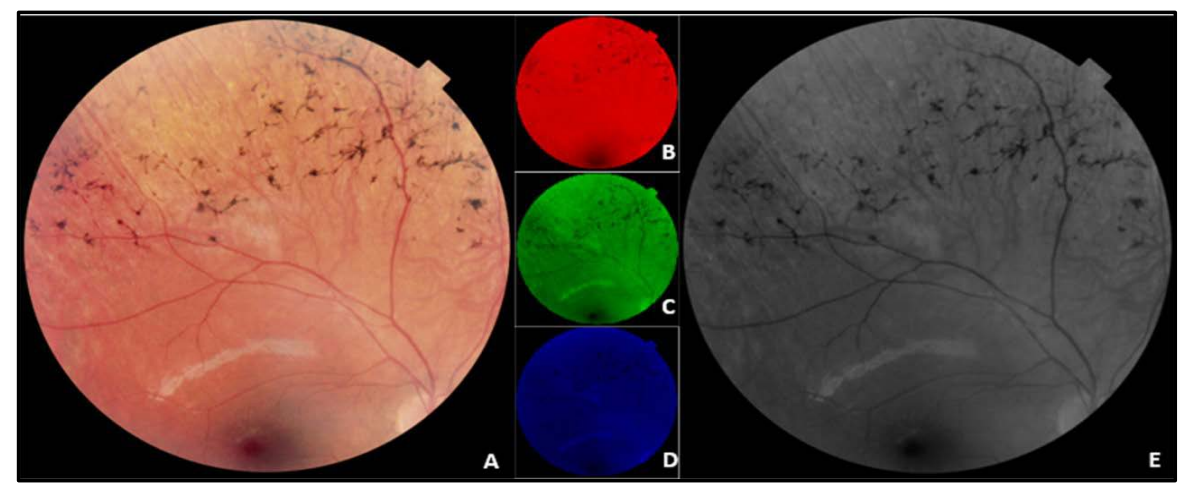

Figure 11. (A) Originalfundus image of a healthy eye. (B) Red Image. (C) Green image. (D) Blue image. (E) Image 8-C after a low-pass filter was applied, resulting in the obtaining of the grayscale image. 


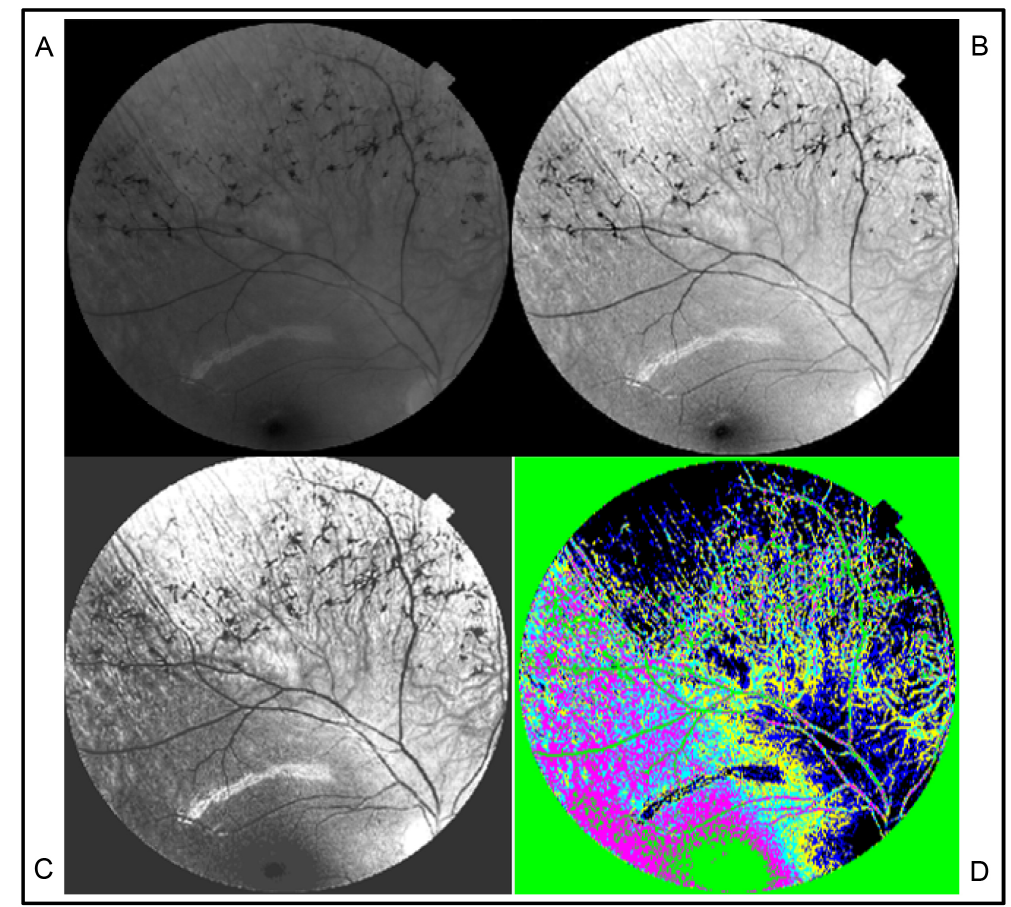

Figure 12. (A) Image with bottom-hat filter application. (B) Contrast enhancement to image 12-A. (C) Histogram equalization with 16 shades of gray to image 12-B. (D) False color method applied to image 12-C.

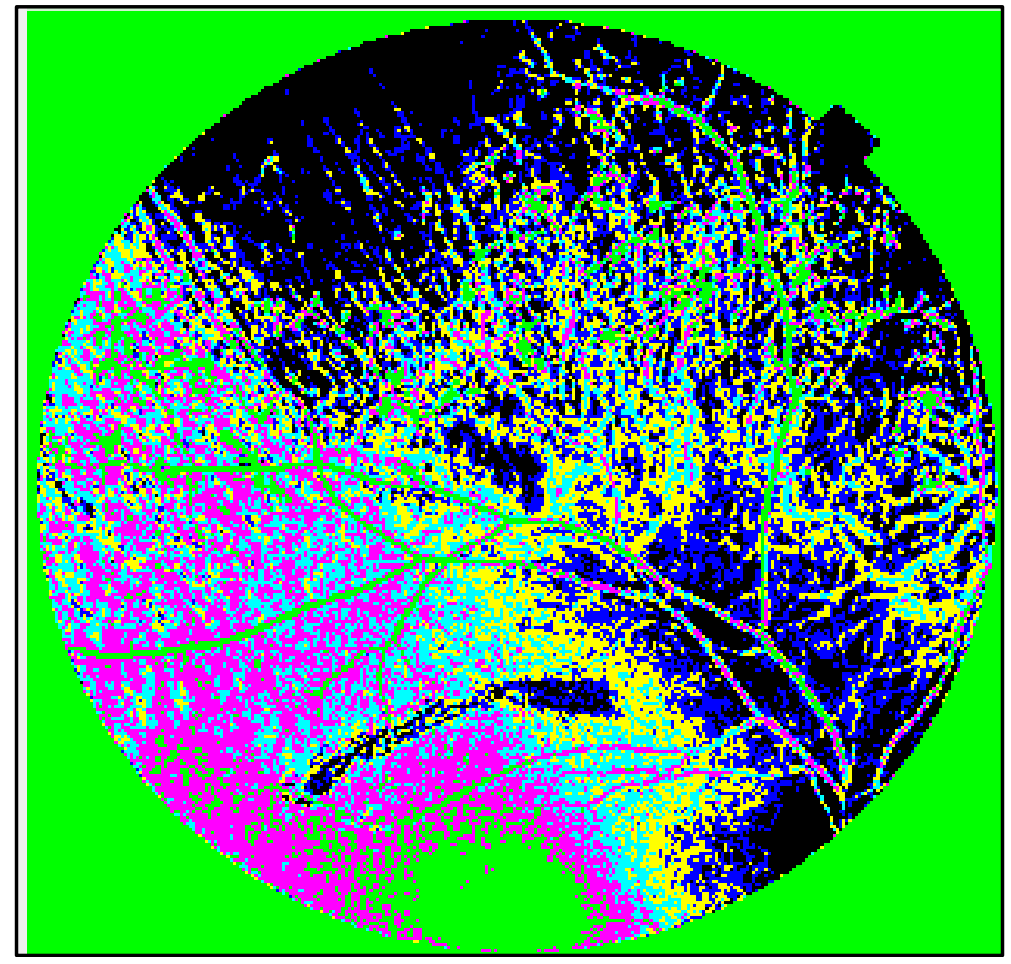

Figure 13. False color method applied to a retinitis pigmentosa fundus image. 


\section{DISCUSSION}

The use of different methods and filters to obtain the differences between two images allows observing whether the patient is developing a degenerative condition that can lead to vision loss. This study using the False Color Method let observe the choroidal vasculature from a fundus image in order to find details unnoticeable for the naked eye, thus approaching to oxygen saturation levels as reported by Jóna Valgerður Kristjánsdóttir [3]. Now then, Figure 14 displays the oxygen saturation, where the red color represents the highest saturation, the green color average saturation, and the purple color null saturation.

The False Color Method was applied in the same image reported in [3] in order to make the relevant comparisons, shown in Figure 15. Reader can see that the red color is the maximum saturation of oxygen, the green color is the mean saturation and the blue/purple color is a null saturation, analogous like in the aforementioned study.

The False Color Method in Figure 15 displays more clearly the choroidal vasculature and the retinal vessels in Figure 14, where said complex vasculature is not easily noticeable. However, in Figure 15 there is more noise, thus considerably affecting the resulting image.

Figure 16 presents an angiography fundus image with injected fluoresce in from [4]. The image in Figure 16(A) comes from a patient with eye ischemia, in other hand Figure 16(B) shows the same case enhanced with the proposed method. We find in Figure 16(B) color differences within vasculature structure, not clearly visible in Figure 16(A). However, a future research could measure possible oxygenation difference levels.

Figure 17(A) shows the choroidal structure of a healthy eye. Now then, Figure 17(B) displays the angiography of the choroidal structure by applying the proposed method.

Figure 18(A) shows the fundus image of an eye with Retinitis Pigmentosa. After applying the False Color Method, we obtained the resulting image displayed in Figure 18(B).

Please note that Figure 16(A), Figure 17(A), and Figure 18(A) correspond to fundus images obtained with OPTOS' Ultra-widefield Technology (UWF) from an OPTOS California icg OCT [4].

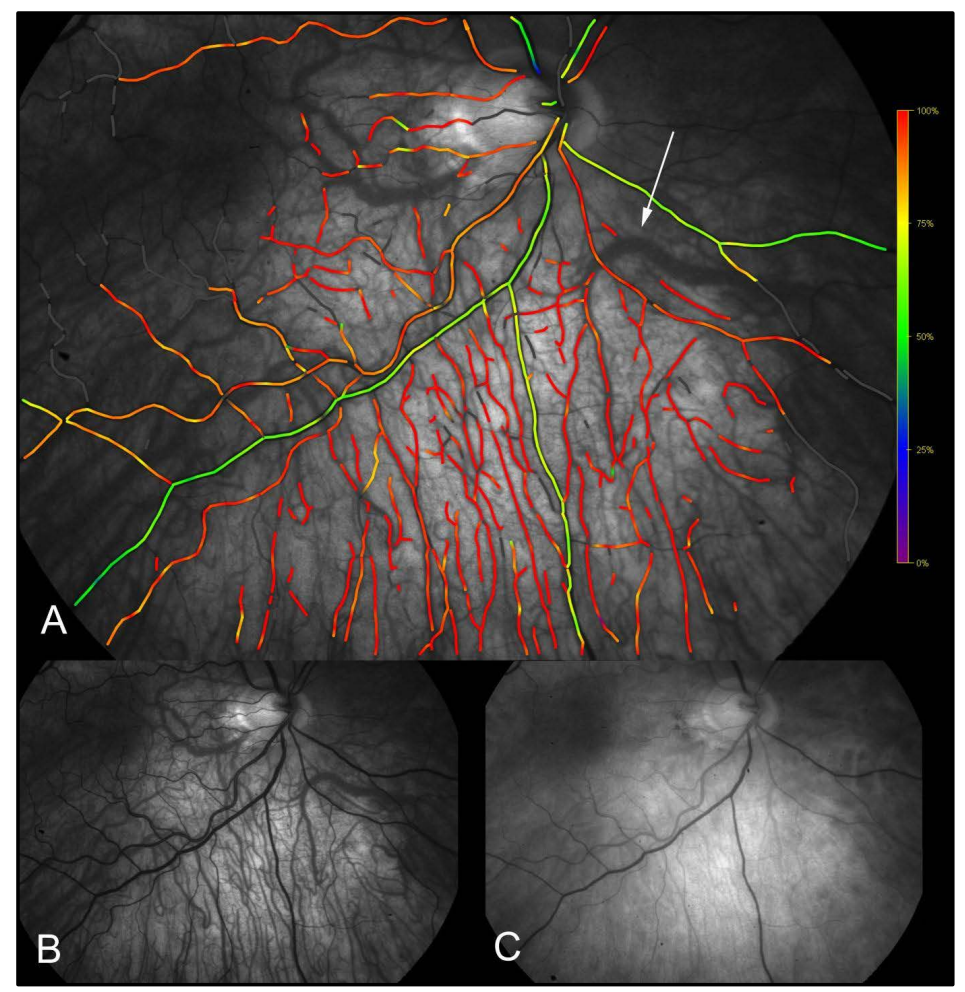

Figure 14. Choroidal oximetry of the eye. 


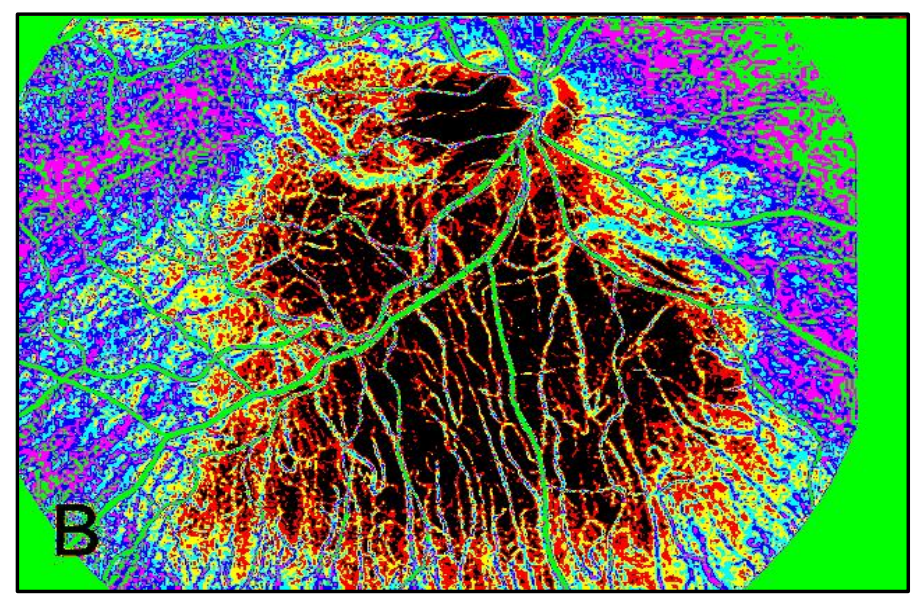

Figure 15. False color method applied to Figure 14(B).

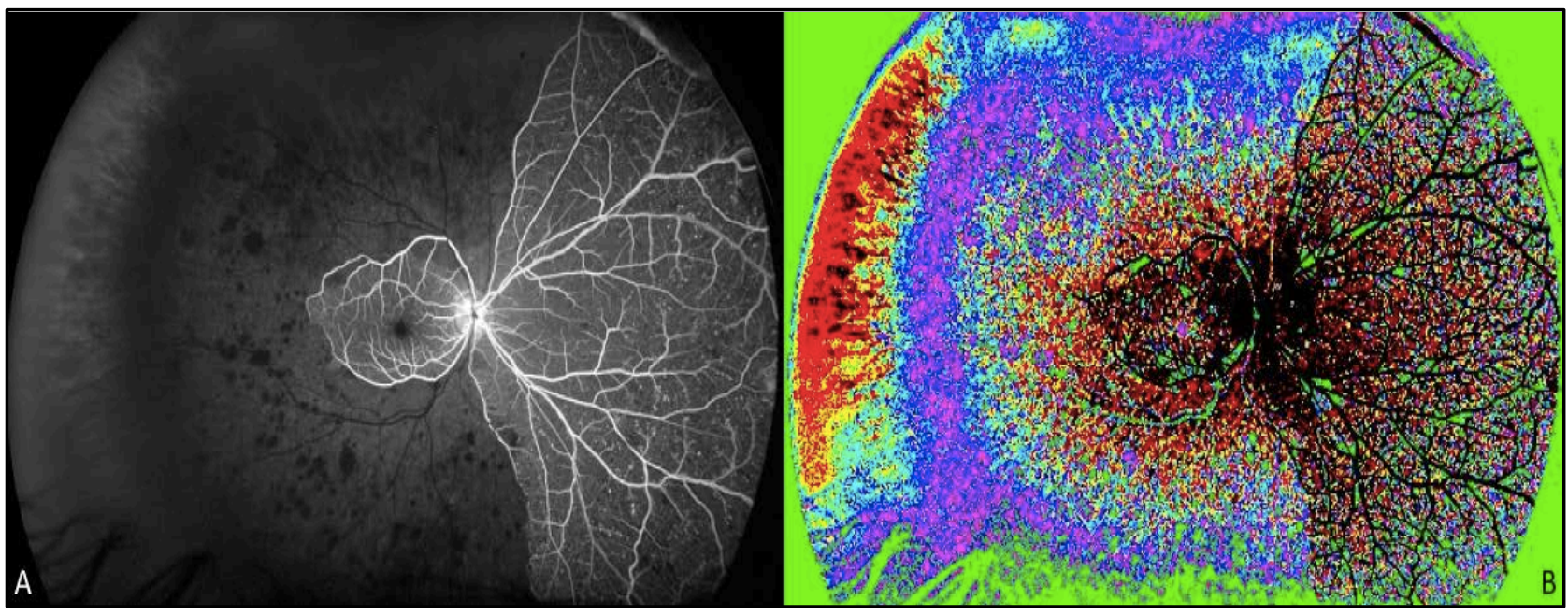

Figure 16. (A) Fundus image of an eye with eye ischemia. (B) False color method applied to the fundus image.

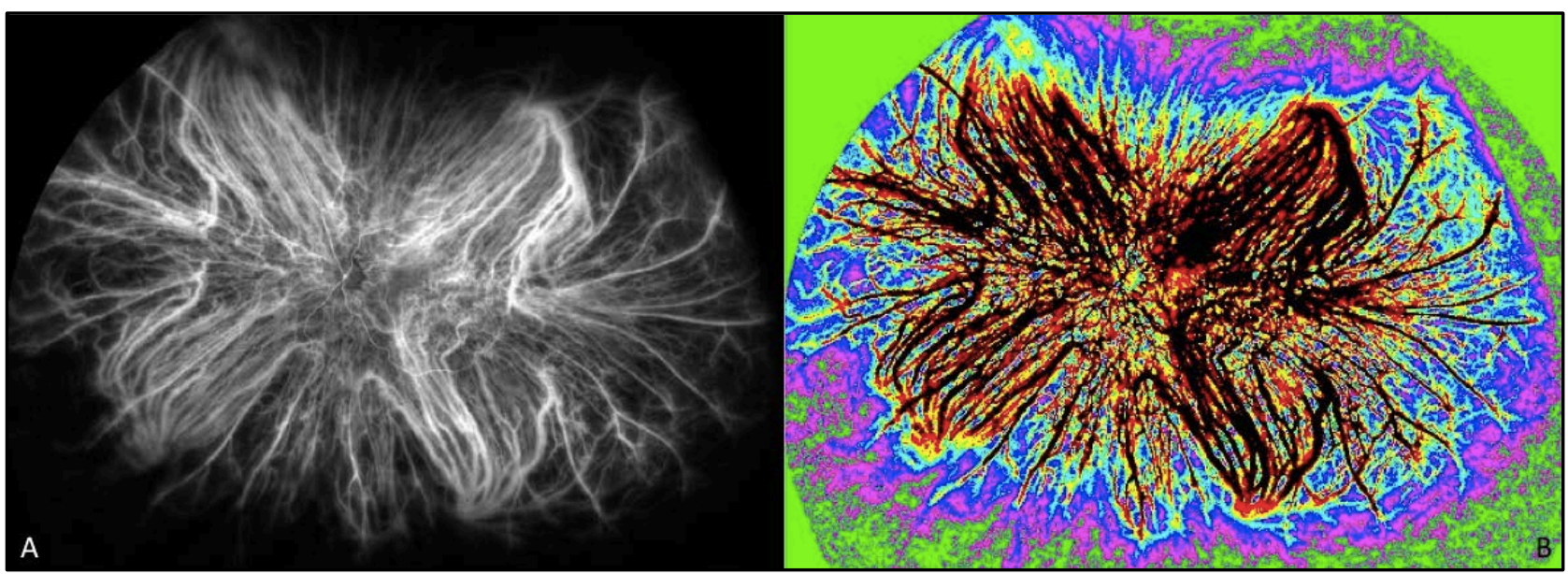

Figura 17. (A) Fundus image of the choroidal structure. (B) False color method applied to the choroid of a health eye. 


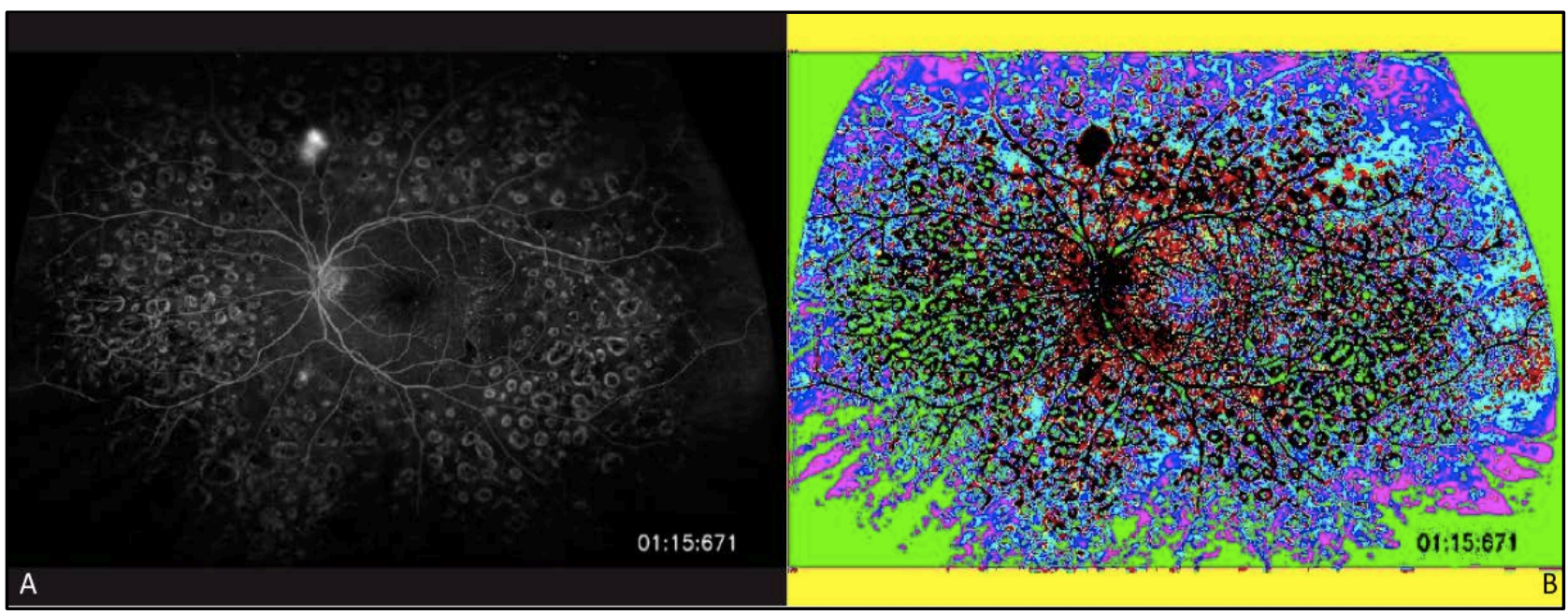

Figure 18. False color method applied to fundus image with retinits pigmentosa.

Finally, due to the absorptivityis inverse at $600 \mathrm{~nm}$ with respect to $570 \mathrm{~nm}$, the algorithm presented was modified to obtain images with inversed colors (as can be seen in from Figures 15-18). These modifications changed the hues that will be assigned to the shades of grey: the values that are closest to black will be assigned a green color and, if the intensity closely resembles white it will be assigned a black color. These images, reported by OPTOS [4] from patients injected with fluorescein solution to pigment the blood and to obtain the fundus laser images with different wavelengths (532 nm for green laser, $635 \mathrm{~nm}$ for red laser, and $488 \mathrm{~nm}$ for blue laser).

\section{CONCLUDING REMARKS}

The proposed method allows enhancing OCT images from either conventional, angiography OCT or laser OCT in order to compare any changes in sequential OCT images. The algorithm detects minimal changes within the structure (i.e. one pixel change, as shown in Figure 1). Figure 8(E) displays the enhancement of the original OCT image. Additionally, in Figure 10 and Figure 13 we show evident differences obtained using the proposed method. As a first approach, the vasculature of the healthy eye is red. However, Figure 13 exposes an eye with Retinitis Pigmentosa showing a high vasculature detail, suppose, with different oxygenation levels that must be measured in a future research as well as a more detailed study of Retinitis Pigmentosa physiopathology.

On the other hand, the method proposed in this work can perform an image study with a single image obtained from any OCT. We concluded that using the False Color Method and the Bottom-Hat filter gives out results similar to those reported by Jóna Valgerður Kristjánsdóttir [3]. Our initial results require a more accurate approach on the color scale.

\section{ACKNOWLEDGEMENTS}

Davila-Iniesta et al. thank CONACyT and Instituto Politecnico Nacional for financial support throughout the making of this work.

\section{CONFLICTS OF INTEREST}

The authors declare that they have no conflicts of interest.

\section{REFERENCES}

1. Graue-Wiechers E. and Graue-Hernandez E. (2009) Ophthalmology in the Practice of General Medicine. 3rd 
Edition, McGraw Hill, Mexico.

2. Not Without My Glasses (2018) Visual Health in Mexico: Half Population Needs Glasses. http://www.nosinmisgafas.info/blog/salud-visual/salud-visual-mexico

3. Kristjánsdóttir, J.V. (2014) Chroroidal and Retinal Oximetry. Master's Thesis, University of Iceland, Reykjavik, Iceland.

4. OPTOS (2018) California icg Was Developed for Retinal Specialists to Optimize Management of AMD, Uveitic Conditions and Other Choroidal Pathology. https://www.optos.com/en/products/california-icg/

5. Fernandez-Revuelta, A. (2012) Fundus Exploration Technique. Actualización en Medicina de Familia, 8, 383-387.

6. Valls-Ferran, M.I., Clement-Corral, A. and Puertas-Bordallo, D. (2018) Fundus. Pediatr Integral, 12, 58.E1-58.E7.

7. Zijlstra, W.G., Buursma, A. and van-Assendelft, O.W. (2000) Visible and Near Infrared Absorption Spectra of Human and Animal Haemoglobin. Determination and Application. 1st Edition, CRC Press, Utrecht, The Netherlands.

8. Manzanaro, G. (2004) Photography of Fundus with Filters. Boletín de la Sociedad de Oftalmología de Madrid, 44, 1-9. https://doi.org/10.1097/IAE.0000000000000882

9. De-Carlo, T.E., Chin, A.T., Bonini-Filho, M.A., Adhi, M., Branchini, L., Salz, D.A.,Baumal, C.R., Crawford, C., Reichel, E., Witkin, A.J., Duker, J.S. and Waheed, N.K. (2015). Detection of Microvascular Changes in Eyes of Patients with Diabetes but Not Clinical Diabetic Retinopathy Using Optical Coherence Tomography Angiography. RETINA, 35, 2364-2370.

10. Gao, S.S., Lui, G., Huang, D. and Jia, Y. (2015) Optimization of the Split-Sprectrum Amplitude-Decorrelation Angiography Algorithm on a Spectral Optical Coherence Tomography System. Optics Letters, 40, 2305-2308. https://doi.org/10.1364/OL.40.002305

11. Holm, S.P. (2014) Optical Imaging of Retinal Blood Flow: Studies in Automatic Vessel Extraction, Alignment, and Driven Changes in Vessel Oximetry. Doctoral Dissertation, University of Manchester, Manchester, United Kingdom.

12. Mahmudi, T., Kafieh, R., Rabbani, H., Mehri-dehnavi, A. and Akhlagi, M. (2014) Comparison of Macular OCTs in Right and Left Eyes of Normal People. Medical Imaging, 9038, 90381W-1-90381W-6.

https://doi.org/10.1117/12.2044046 